\title{
SPACECRAFT COORDINATION CONTROL USING PID+ BACKSTEPPING
}

\author{
Raymond Kristiansen * Thomas R. Krogstad ${ }^{* *}$ \\ Per J. Nicklasson* Jan T. Gravdahl ** \\ * Department of Computer Science, Electrical Engineering and \\ Space technology, Narvik University College, N-8505 Narvik, \\ Norway \\ ** Department of Engineering Cybernetics, Norwegian \\ University of Science and Technology, N-7034 Trondheim, \\ Norway
}

\begin{abstract}
In this paper we present a PID+ solution to the problem of relative translational and rotational tracking, using the concept of integrator backstepping. By using an integrator augmentation technique, we include integral effect in the controller to account for the unknown orbital perturbations. The controller solution utilizes the quaternion representation to achieve a shorter rotation path on commanded attitude changes, and the equilibrium points in the closed-loop system are proved to be uniformly exponentially stable. Finally, simulation results are presented to show the controller performance.
\end{abstract}

Keywords: Spacecraft formation, integrator backstepping, PID+ control

\section{INTRODUCTION}

\subsection{Background}

Synchronization, coordination and cooperative control are new and promising trends within mechanical systems technology. Replacing complex single units with several simpler and less expensive agents makes it possible to achieve larger operational areas with greater flexibility and performance. In the space industry, the concept makes the way for new and better applications, such as improved monitoring of the Earth and its surrounding atmosphere, geodesy, deep-space imaging and exploration and even in-orbit spacecraft servicing and maintenance. However, the advantages of using spacecraft formations come at a cost of increased control complexity and technological challenges. Formation flying introduces a control problem with strict and time-varying boundaries on spacecraft reference trajectories, and requires detailed knowledge and tight control of relative distances and velocities for participating spacecraft.

\subsection{Previous work}

Synchronized control of relative attitude in spacecraft formations has received increased attention over the last years. From the first models of relative translation in circular orbits presented in Hill (1878); Clohessy and Wiltshire (1960), later achievements include nonlinear models of coupled translational and rotational motion in Wang and Hadaegh (1996); Pan and Kapila (2001). State feedback tracking control laws for relative position and attitude were developed in Wang and Hadaegh (1996); Wang et al. (1999), and these solutions were proved to result in exponentially stable equilibrium points in the closed loop system. In Pan and Kapila (2001), a nonlinear tracking controller for both relative position and attitude was presented, including an adaptation law to account for unknown mass and inertia parameters of the spacecraft. The controller ensures global asymptotic convergence of position and velocity errors, and the stability result was proved using a Lyapunov framework and standard signal-chasing arguments. Semiglobal asymptotic convergence of relative position and attitude er- 
rors was proved in Wong et al. (2005) for an adaptive output feedback controller using relative position only, tracing the steps of Pan and Kapila (2001).

\subsection{Contribution}

In this paper, we present a PID+ tracking controller for relative translation and rotation in a leader-follower spacecraft formation, derived using integrator backstepping. The controller is an extension of earlier works in Kristiansen et al. (2006b) on relative attitude control, where the equilibrium points in the closedloop system were proved to be uniformly asymptotically stable (UAS). These latter results were however based on an assumption of known orbital perturbations, which is seldom the case. In this paper, we extend the solution to both relative translation and rotation, and relax the latter assumption to unknown, but constant, perturbations. By using an integrator augmentation technique ( $c f$. Fossen (2002)), we include integral effect in the controller to account for the unknown orbital perturbations. Hence, the equilibrium points in the closed-loop system are proved to be UES.

The rest of the paper is organized as follows: Section 2 defines the different reference frames used and presents the mathematical models of relative attitude dynamics and kinematics in a leader-follower spacecraft formation. The controller design is performed in Section 3, and simulation results of a system with the derived controller are presented in Section 4. Concluding remarks are given in Section 5.

\section{MODELLING}

In this section, we describe the different coordinate frames, together with the necessary coordinate transformations, before we present models of spacecraft relative translation and rotation.

\subsection{Cartesian coordinate frames}

The coordinate reference frames used throughout the paper aredefined as follows:

Earth Centered Inertial (ECI) frame: This frame is denoted $\mathcal{F}_{i}$, and its origin is located in the center of the Earth. Its $z$ axis is directed along the rotation axis of the Earth towards the celestial north pole, the $x$ axis coincide with the vernal equinox vector towards the vernal point, and finally the direction of the $y$ axis completes a right handed orthogonal frame.

Leader orbit reference frame: The leader orbit frame, denoted $\mathcal{F}_{l}$, has its origin located in the center of mass of the leader spacecraft. The $\mathbf{e}_{r}$ axis in the frame coincide with the vector $\mathbf{r}_{l}$ from the center of the Earth to the spacecraft, and the $\mathbf{e}_{h}$ axis is parallel to the orbital angular momentum vector, pointing in the orbit normal direction. The $\mathbf{e}_{\theta}$ axis completes the right-handed orthogonal frame. The basis vectors of the frame can be defined as

$$
\mathbf{e}_{r}:=\frac{\mathbf{r}_{l}}{r_{l}}, \quad \mathbf{e}_{\theta}:=\mathbf{e}_{h} \times \mathbf{e}_{r} \quad \text { and } \quad \mathbf{e}_{h}:=\frac{\mathbf{h}}{h},
$$

where $\mathbf{h}=\mathbf{r}_{l} \times \dot{\mathbf{r}}_{l}$ is the angular momentum vector of the orbit, $h=|\mathbf{h}|$ and $r_{l}=\left|\mathbf{r}_{l}\right|$.

Follower orbit reference frame: This frame has its origin in the center of mass of the follower spacecraft, and is denoted $\mathcal{F}_{f}$. The vector pointing from the center of the Earth to frame origin is denoted $\mathbf{r}_{f}$. It is spec-

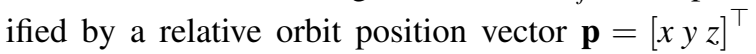
expressed in $\mathcal{F}_{l}$ frame components, and the frame unit vectors align with the basis vectors of $\mathcal{F}_{l}$. Accordingly,

$$
\mathbf{p}=\mathbf{r}_{f}-\mathbf{r}_{l}=x \mathbf{e}_{r}+y \mathbf{e}_{\theta}+z \mathbf{e}_{h} .
$$

Body reference frames: For both the leader and the follower spacecraft, body reference frames are defined and denoted $\mathcal{F}_{b l}$ and $\mathcal{F}_{b f}$, respectively. These frames have, similar to the orbit frame, the origin located in the center of mass of the respective spacecraft, but the basis vectors are fixed in the spacecraft body and coincide with its principal axis of inertia.

\subsection{Body frame rotation}

The rotation matrix describing rotations from an orbit frame to a body frame can be described by

$$
\mathbf{R}_{o}^{b}=\left[\mathbf{c}_{1} \mathbf{c}_{2} \mathbf{c}_{3}\right]=\mathbf{I}+2 \eta \mathbf{S}(\varepsilon)+2 \mathbf{S}^{2}(\varepsilon)
$$

where the elements $\mathbf{c}_{i}$ are directional cosines, and $\mathbf{q}=\left[\eta \varepsilon^{T}\right]^{T}$ are the Euler parameters, which satisfy

$$
\eta^{2}+\varepsilon^{\top} \varepsilon=1 .
$$

The matrix $\mathbf{S}(\cdot)$ is the cross product operator given by

$$
\mathbf{S}(\varepsilon)=\varepsilon \times=\left[\begin{array}{ccc}
0 & -\varepsilon_{z} & \varepsilon_{y} \\
\varepsilon_{z} & 0 & -\varepsilon_{x} \\
-\varepsilon_{y} & \varepsilon_{x} & 0
\end{array}\right]
$$

when $\varepsilon=\left[\begin{array}{lll}\varepsilon_{x} & \varepsilon_{y} & \varepsilon_{z}\end{array}\right]^{T}$. The inverse rotation is given by the complex conjugate of $\mathbf{q}$ as $\overline{\mathbf{q}}=\left[\eta-\varepsilon^{T}\right]^{T}$.

\subsection{Relative translation}

The fundamental differential equation of the two-body problem can be found as (cf. Battin (1999))

$$
\frac{d^{2} \mathbf{r}}{d t^{2}}+\frac{\mu}{r^{3}} \mathbf{r}=\mathbf{0}
$$

where $\mathbf{r}=\mathbf{r}_{2}-\mathbf{r}_{1}$ is the relative position of masses $m_{1}$ and $m_{2}$ with respective ECI position vectors $\mathbf{r}_{1}$ and $\mathbf{r}_{2}$, and $\mu=G\left(m_{1}+m_{2}\right)$ with $G$ as the universal constant of gravity. This equation can be generalized to include force terms due to aerodynamic disturbances, gravitational forces from other bodies, solar radiation, magnetic fields and so on. In addition, it can be augmented to include control input vectors from onboard actuators. Accordingly, (6) can be expressed as 


$$
\ddot{\mathbf{r}}_{s}=-\frac{\mu}{r_{s}^{3}} \mathbf{r}_{s}+\frac{\mathbf{f}_{d s}}{m_{s}}+\frac{\mathbf{f}_{a s}}{m_{s}}
$$

where $\mathbf{f}_{d s} \in \mathbb{R}^{3}$ is the orbital perturbation term due to external effects and $\mathbf{f}_{a s} \in \mathbb{R}^{3}$ is the actuator force. The superscript/subscript $s$ is used in general to denote the spacecraft in question, so $s=l, f$ for the leader and follower spacecraft, respectively. In addition, spacecraft masses are assumed to be small relative to the mass of the Earth $M_{e}$, so $\mu \approx G M_{e}$. Using the true anomaly $v$ of the leader spacecraft, and denoting relative velocity as $\mathbf{v}=\dot{\mathbf{p}}$, the nonlinear position dynamics can be represented in the $\mathcal{F}_{l}$ frame on the form ( $c f$. Yan et al. (2000),Kristiansen et al. (2007))

$$
m_{f} \dot{\mathbf{v}}+\mathbf{C}_{t}(\dot{\mathrm{v}}) \mathbf{v}+\mathbf{D}_{t}\left(\dot{\mathrm{v}}, \ddot{\mathbf{v}}, r_{f}\right) \mathbf{p}+\mathbf{n}_{t}\left(r_{l}, r_{f}\right)=\mathbf{F}_{a}+\mathbf{F}_{d}
$$

where

$$
\mathbf{C}_{t}(\dot{\mathrm{v}})=2 m_{f} \dot{\mathrm{v}}\left[\begin{array}{ccc}
0 & -1 & 0 \\
1 & 0 & 0 \\
0 & 0 & 0
\end{array}\right] \in S S(3)
$$

is a skew-symmetric Coriolis-like matrix,

$$
\mathbf{D}_{t}\left(\dot{v}, \ddot{v}, r_{f}\right) \mathbf{p}=m_{f}\left[\begin{array}{ccc}
\frac{\mu}{r_{f}^{3}}-\dot{v}^{2} & -\ddot{v} & 0 \\
\ddot{v} & \frac{\mu}{r_{f}^{3}}-\dot{v}^{2} & 0 \\
0 & 0 & \frac{\mu}{r_{f}^{3}}
\end{array}\right] \mathbf{p}
$$

may be viewed as a time-varying potential force, and

$$
\mathbf{n}_{t}\left(r_{l}, r_{f}\right)=m_{f} \mu\left[\frac{r_{l}}{r_{f}^{3}}-\frac{1}{r_{l}^{2}}, 0,0\right]^{\top} .
$$

The composite perturbation force $\mathbf{F}_{d}$ and the relative control force $\mathbf{F}_{a}$ are given by

$$
\mathbf{F}_{d}=\mathbf{f}_{d f}-\frac{m_{f}}{m_{l}} \mathbf{f}_{d l} \quad \mathbf{F}_{a}=\mathbf{f}_{a f}-\frac{m_{f}}{m_{l}} \mathbf{f}_{a l} .
$$

\subsection{Relative rotation}

The time derivative of a matrix $\mathbf{R}_{b}^{a}$ as in (3) can according to Egeland and Gravdahl (2002) be written

$$
\dot{\mathbf{R}}_{b}^{a}=\mathbf{S}\left(\omega_{a, b}^{a}\right) \mathbf{R}_{b}^{a}=\mathbf{R}_{b}^{a} \mathbf{S}\left(\omega_{a, b}^{b}\right)
$$

where $\omega_{a, b}^{b} \in \mathbb{R}^{3}$ is the angular velocity of frame $\mathcal{F}_{b}$ relative to frame $\mathcal{F}_{a}$ decomposed in frame $\mathcal{F}_{b}$ and $\mathbf{S}(\cdot)$ is the cross product operator described in (5). The kinematic equations for a spacecraft in its orbit frame can be found from (13) as

$$
\dot{\mathbf{q}}_{s}=\mathbf{T}\left(\mathbf{q}_{s}\right) \omega_{s, s b}^{s b}, \quad \mathbf{T}\left(\mathbf{q}_{s}\right)=\frac{1}{2}\left[\begin{array}{c}
-\varepsilon_{s}^{T} \\
\eta_{s} \mathbf{I}+\mathbf{S}\left(\varepsilon_{s}\right)
\end{array}\right]
$$

where $\omega_{s, s b}^{s b} \in \mathbb{R}^{3}$ is the angular velocity of the spacecraft body frame relative to the orbit frame, referenced in the body frame. Moreover, with the assumptions of rigid body movement, the dynamical model of a spacecraft can be found from Euler's momentum equation as ( $c f$. Battin (1999))

$$
\begin{aligned}
\mathbf{J}_{s} \dot{\omega}_{i, s b}^{s b} & =-\mathbf{S}\left(\omega_{i, s b}^{s b}\right) \mathbf{J}_{s} \omega_{i, s b}^{s b}+\tau_{d s}^{s b}+\tau_{a s}^{s b} \\
\omega_{s, s b}^{s b} & =\omega_{i, s b}^{s b}+\omega_{o} \mathbf{c}_{2}
\end{aligned}
$$

where $\mathbf{J}_{s}$ is the spacecraft inertia matrix and $\omega_{i, s b}^{s b} \in \mathbb{R}^{3}$ is the angular velocity of the spacecraft body frame relative to the inertial frame, expressed in the body frame. The scalar parameter $\omega_{o}$ is the orbit angular velocity, $\tau_{d}^{s b} \in \mathbb{R}^{3}$ is the perturbation torque, $\tau_{a}^{s b} \in \mathbb{R}^{3}$ is the actuator torque, and $\mathbf{c}_{2} \in \mathbb{R}^{3}$ is the directional cosine vector from (3). Further, by expressing the relations in (14) and (15)-(16) for both the leader and the follower spacecraft, and utilising the quaternion product defined in Egeland and Gravdahl (2002) as

$$
\mathbf{q}=\mathbf{q}_{f} \otimes \overline{\mathbf{q}}_{l}:=\left[\begin{array}{c}
\eta_{f} \eta_{l}+\varepsilon_{f}^{T} \varepsilon_{l} \\
\eta_{l} \varepsilon_{f}-\eta_{f} \varepsilon_{l}-\mathbf{S}\left(\varepsilon_{f}\right) \varepsilon_{l}
\end{array}\right]
$$

the relative attitude kinematics and dynamics can be expressed as

$$
\dot{\mathbf{q}}=\left[\begin{array}{c}
\dot{\eta} \\
\dot{\varepsilon}
\end{array}\right]=\mathbf{T}(\mathbf{q}) \omega
$$

where

$$
\omega=\omega_{i, f b}^{f b}-\mathbf{R}_{l b}^{f b} \omega_{i, l b}^{l b}
$$

is the relative angular velocity between the leader body reference frame and the follower body reference frame. Moreover, from (19) the relative attitude dynamics can be expressed as

$$
\mathbf{J}_{f} \dot{\boldsymbol{\omega}}=\mathbf{J}_{f} \dot{\omega}_{i, f b}^{f b}-\mathbf{J}_{f} \mathbf{S}\left(\omega_{i, l b}^{f b}\right) \omega-\mathbf{J}_{f} \mathbf{R}_{l b}^{f b} \dot{\omega}_{i, l b}^{l b}
$$

where (13) and the facts that $\omega_{l b, f b}^{f b}=\omega$ and $\mathbf{S}(\mathbf{a}) \mathbf{b}=$ $-\mathbf{S}(\mathbf{b}) \mathbf{a}$ have been used. Insertion of (15), evaluated for both the leader and follower, into (20) results in (cf. Kristiansen et al. (2007))

$$
\mathbf{J}_{f} \dot{\boldsymbol{\omega}}+\mathbf{C}_{r}(\omega) \omega+\mathbf{n}_{r}(\omega)=\Upsilon_{d}+\Upsilon_{a}
$$

where

$$
\begin{array}{r}
\mathbf{C}_{r}(\omega)=\mathbf{J}_{f} \mathbf{S}\left(\mathbf{R}_{l b}^{f b} \omega_{i, l b}^{l b}\right)+\mathbf{S}\left(\mathbf{R}_{l b}^{f b} \omega_{i, l b}^{l b}\right) \mathbf{J}_{f} \\
-\mathbf{S}\left(\mathbf{J}_{f}\left[\omega+\mathbf{R}_{l b}^{f b} \omega_{i, l b}^{l b}\right]\right)
\end{array}
$$

is a skew-symmetric matrix, $\mathbf{C}_{r}(\omega) \in S S(3)$,

$$
\begin{aligned}
\mathbf{n}_{r}(\omega)=\mathbf{S}\left(\mathbf{R}_{l b}^{f b} \omega_{i, l b}^{l b}\right) \mathbf{J}_{f} \mathbf{R}_{l b}^{f b} \omega_{i, l b}^{l b} \\
\\
\quad-\mathbf{J}_{f} \mathbf{R}_{l b}^{f b} \mathbf{J}_{l}^{-1} \mathbf{S}\left(\omega_{i, l b}^{l b}\right) \mathbf{J}_{l} \omega_{i, l b}^{l b}
\end{aligned}
$$

is a nonlinear term, and

$\Upsilon_{d}=\tau_{d f}^{f b}-\mathbf{J}_{f} \mathbf{R}_{l b}^{f b} \mathbf{J}_{l}^{-1} \tau_{d l}^{l b}, \quad \Upsilon_{a}=\tau_{a f}^{f b}-\mathbf{J}_{f} \mathbf{R}_{l b}^{f b} \mathbf{J}_{l}^{-1} \tau_{a l}^{l b}$

are the relative perturbation torques and relative actuator torques, respectively.

\subsection{Total model}

To write the total 6DOF model of relative translation and rotation in the spacecraft formation, we define the state vectors

$$
\mathbf{x}_{1}:=\left[\mathbf{p}^{\top} \mathbf{q}^{\top}\right]^{\top} \quad \text { and } \quad \mathbf{x}_{2}:=\left[\mathbf{v}^{\top} \omega^{\top}\right]^{\top} .
$$


Based on (8) and (21), the total model of the relative translational and rotational motion between the leader and the follower spacecraft can now be expressed

$$
\begin{aligned}
& \dot{\mathbf{x}}_{1}=\Lambda\left(\mathbf{x}_{1}\right) \mathbf{x}_{2} \\
& \mathbf{M}_{f} \dot{\mathbf{x}}_{2}+\mathbf{C}(\dot{\mathrm{v}}, \omega) \mathbf{x}_{2}+\mathbf{D}\left(\dot{\mathrm{v}}, \ddot{v}, r_{f}\right) \mathbf{x}_{1}+ \\
& \mathbf{n}\left(\omega, r_{l}, r_{f}\right)=\mathbf{U}+\mathbf{W}
\end{aligned}
$$

where

$$
\Lambda\left(\mathbf{x}_{1}\right)=\left[\begin{array}{cc}
\mathbf{I} & \mathbf{0} \\
\mathbf{0} & \mathbf{T}(\mathbf{q})
\end{array}\right] \quad \text { and } \quad \mathbf{M}_{f}=\left[\begin{array}{cc}
m_{f} \mathbf{I} & \mathbf{0} \\
\mathbf{0} & \mathbf{J}_{f}
\end{array}\right]
$$

are the coupling term between the first and second order dynamics, and a symmetric positive definite matrix containing the mass and moments of inertia of the follower, respectively;

$$
\mathbf{C}(\dot{\mathrm{v}}, \omega)=\left[\begin{array}{cc}
\mathbf{C}_{t}(\dot{\mathrm{v}}) & \mathbf{0} \\
\mathbf{0} & \mathbf{C}_{r}(\boldsymbol{\omega})
\end{array}\right] \in S S(6)
$$

is the skew-symmetric Coriolis-like matrix,

$$
\mathbf{D}\left(\dot{\mathrm{v}}, \ddot{v}, r_{f}\right) \mathbf{x}_{1}=\left[\begin{array}{cc}
\mathbf{D}_{t}\left(\dot{\mathrm{v}}, \ddot{\mathrm{v}}, r_{f}\right) & \mathbf{0} \\
\mathbf{0} & \mathbf{0}
\end{array}\right] \mathbf{x}_{1}
$$

is the time-varying potential force term,

$$
\mathbf{n}\left(\omega, r_{l}, r_{f}\right)=\left[\begin{array}{c}
\mathbf{n}_{t}\left(r_{l}, r_{f}\right) \\
\mathbf{n}_{r}(\omega)
\end{array}\right]
$$

is the composite nonlinear term, and finally

$$
\mathbf{U}=\left[\mathbf{F}_{a}^{\top}, \Upsilon_{a}^{\top}\right]^{\top} \quad \text { and } \quad \mathbf{W}=\left[\mathbf{F}_{d}^{\top}, \Upsilon_{d}^{\top}\right]^{\top}
$$

contains the relative input forces and orbital perturbations, respectively.

\section{CONTROLLER DESIGN}

Having established the 6DOF mathematical model of relative motion in a leader-follower formation, we now present a solution to the control problem.

\subsection{Integrator backstepping}

The control problem is to design a controller that makes the state $\mathbf{x}_{1}$ converge to and proceed to track a time-varying smooth trajectory $\mathbf{x}_{d}(t)$. The desired trajectory can be specified as

$$
\mathbf{x}_{d 1}=\left[\mathbf{p}_{d}^{\top}, \mathbf{q}_{d}^{\top}\right]^{\top} \quad \mathbf{x}_{d 2}=\left[\mathbf{v}_{d}^{\top}, \omega_{d}^{\top}\right]^{\top}
$$

so that $\dot{\mathbf{x}}_{d 1}=\Lambda\left(\mathbf{x}_{d 1}\right) \mathbf{x}_{d 2}$. To exploit the redundancy in the quaternion parameter representation ( $c f$. Kristiansen et al. (2006b)), we define the state error as

$$
\mathbf{e}:=[\tilde{\mathbf{p}}, 1-|\tilde{\eta}|, \tilde{\varepsilon}]
$$

where $\tilde{\mathbf{p}}=\mathbf{p}-\mathbf{p}_{d}$ is the translation error, and the rotation error $\tilde{\mathbf{q}}=\left[\tilde{\eta} \tilde{\varepsilon}^{\top}\right]^{\top}$ is given from the quaternion product

$$
\tilde{\mathbf{q}}=\mathbf{q} \otimes \overline{\mathbf{q}}_{d}=\left[\begin{array}{c}
\eta \eta_{d}+\varepsilon^{\top} \varepsilon_{d} \\
\eta_{d} \varepsilon-\eta \varepsilon_{d}-\mathbf{S}(\varepsilon) \varepsilon_{d}
\end{array}\right] .
$$

Perfect trajectory tracking can be expressed as

$$
\mathbf{x}_{1}(t)=\mathbf{x}_{d 1}(t) \quad \Leftrightarrow \quad \tilde{\mathbf{x}}_{1}(t)=\left[\begin{array}{c}
\tilde{\mathbf{p}} \\
\tilde{\mathbf{q}}
\end{array}\right]=\left[\begin{array}{c}
\mathbf{0} \\
\pm 1 \\
\mathbf{0}
\end{array}\right]
$$

for all $t \geq 0$. Following the notation in (14), it can be shown that

$$
\dot{\tilde{\mathbf{q}}}=\mathbf{T}(\tilde{\mathbf{q}}) \tilde{\omega}=\frac{1}{2}\left[\begin{array}{c}
-\tilde{\boldsymbol{\varepsilon}}^{\top} \\
\tilde{\eta} \mathbf{I}+\mathbf{S}(\tilde{\varepsilon})
\end{array}\right] \tilde{\omega}
$$

with $\tilde{\omega}=\omega-\omega_{d}$. Similarly, it can be shown that

$$
\dot{\mathbf{e}}=\left[\begin{array}{c}
\dot{\tilde{\mathbf{p}}} \\
-\underset{\tilde{\tilde{\varepsilon}}}{\operatorname{sgn}(\tilde{\eta})} \dot{\tilde{\eta}}
\end{array}\right]=\mathbf{G}^{\top}\left(\tilde{\mathbf{x}}_{1}\right) \tilde{\mathbf{x}}_{2}
$$

where

$$
\mathbf{G}^{\top}\left(\tilde{\mathbf{x}}_{1}\right)=\left[\begin{array}{cc}
\mathbf{I} & \mathbf{0} \\
\mathbf{0} & \frac{1}{2}\left[\begin{array}{c}
\operatorname{sgn}(\tilde{\eta}) \tilde{\varepsilon}^{\top} \\
(\tilde{\eta} \mathbf{I}+\mathbf{S}(\tilde{\varepsilon}))
\end{array}\right]
\end{array}\right]
$$

and $\tilde{\mathbf{x}}_{2}=[\tilde{\mathbf{v}}, \tilde{\omega}]$. To include integral effect in the controller to withstand constant unknown perturbations, we define our first backstepping subsystem as

$$
\dot{\mathbf{z}}_{0}:=\mathbf{G e}
$$

where the argument of the matrix $\mathbf{G}\left(\tilde{\mathbf{x}}_{1}\right)$ is left out for readability. Note that

$$
\mathbf{G e}=\mathbf{0} \Leftrightarrow\left[\begin{array}{c}
\tilde{\mathbf{p}} \\
\operatorname{sgn}(\tilde{\eta}) \tilde{\varepsilon}
\end{array}\right]=\mathbf{0}
$$

and the signum function $\operatorname{sgn}(\tilde{\eta})$ is therefore defined nonzero as

$$
\operatorname{sgn}(\tilde{\eta}):=\left\{\begin{aligned}
-1, & \tilde{\eta}<0 \\
1, & \tilde{\eta} \geq 0
\end{aligned}\right.
$$

to avoid a singularity when $\tilde{\eta}=0$. We choose our first virtual control input as the entire integral term;

$$
\mathbf{G e}=\alpha_{0}+\mathbf{z}_{1}
$$

with $\alpha_{0}$ as a stabilizing function and $\mathbf{z}_{1}$ as a new state variable. Moreover, we define our first Lyapunov function candidate as

$$
\begin{aligned}
V_{0} & :=\frac{1}{2} \mathbf{z}_{0}^{\top} \mathbf{z}_{0} \\
\dot{V}_{0} & =\mathbf{z}_{0}^{\top} \dot{\mathbf{z}}_{0}=\mathbf{z}_{0}^{\top} \mathbf{G e}=\mathbf{z}_{0}^{\top}\left(\alpha_{0}+\mathbf{z}_{1}\right) .
\end{aligned}
$$

Choosing the stabilizing function as

$$
\alpha_{0}=-\mathbf{K}_{0} \mathbf{z}_{0}
$$

with $\mathbf{K}_{0}=\mathbf{K}_{0}^{\top}>0$ as controller gain, yields

$$
\begin{aligned}
& \dot{V}_{0}=-\mathbf{z}_{0}^{\top} \mathbf{K}_{0} \mathbf{z}_{0}+\mathbf{z}_{0}^{\top} \mathbf{z}_{1} \\
& \dot{\mathbf{z}}_{0}=-\mathbf{K}_{0} \mathbf{z}_{0}+\mathbf{z}_{1} .
\end{aligned}
$$

From (43) we now get the $\mathbf{z}_{1}$ subsystem, and differentiation and insertion of (38) yields

$$
\dot{\mathbf{z}}_{1}=\tilde{\mathbf{x}}_{2}+\dot{\mathbf{G}} \mathbf{e}-\dot{\alpha}_{0}
$$

since $\mathbf{G G}^{\top}=$ I. A virtual control input for the $\mathbf{z}_{1}$ subsystem is now defined as

$$
\tilde{\mathbf{x}}_{2}:=\alpha_{1}+\mathbf{z}_{2}
$$

together with the Lyapunov function candidate 


$$
\begin{aligned}
V_{1} & :=V_{0}+\frac{1}{2} \mathbf{z}_{1}^{\top} \mathbf{z}_{1} \\
\dot{V}_{1} & =-\mathbf{z}_{0}^{\top} \mathbf{K}_{0} \mathbf{z}_{0}+\mathbf{z}_{1}^{\top}\left(\alpha_{1}+\mathbf{z}_{2}+\mathbf{z}_{0}+\dot{\mathbf{G}} \mathbf{e}-\dot{\alpha}_{0}\right) .
\end{aligned}
$$

Choosing the stabilizing function $\alpha_{1}$ as

$$
\alpha_{1}=-\mathbf{K}_{1} \mathbf{z}_{1}-\mathbf{z}_{0}-\dot{\mathbf{G}} \mathbf{e}+\dot{\alpha}_{0}
$$

where $\mathbf{K}_{1}=\mathbf{K}_{1}^{\top}>0$ is a feedback gain matrix, results in

$$
\begin{aligned}
& \dot{V}_{1}=-\mathbf{z}_{0}^{\top} \mathbf{K}_{0} \mathbf{z}_{0}-\mathbf{z}_{1}^{\top} \mathbf{K}_{1} \mathbf{z}_{1}+\mathbf{z}_{1}^{\top} \mathbf{z}_{2} \\
& \dot{\mathbf{z}}_{1}=-\mathbf{K}_{1} \mathbf{z}_{1}-\mathbf{z}_{0}+\mathbf{z}_{2} .
\end{aligned}
$$

For the final step, (50) is differentiated to express the $\mathbf{z}_{2}$-dynamics as $\dot{\mathbf{z}}_{2}=\dot{\mathbf{x}}_{2}-\dot{\mathbf{x}}_{d 2}-\dot{\alpha}_{1}$ and insertion of (27) results in

$$
\begin{aligned}
\mathbf{M}_{f} \dot{\mathbf{z}}_{2} & =\mathbf{M}_{f} \dot{\mathbf{x}}_{2}-\mathbf{M}_{f} \dot{\mathbf{x}}_{d 2}-\mathbf{M}_{f} \dot{\alpha}_{1} \\
& =\mathbf{U}+\mathbf{W}-\mathbf{C} \mathbf{x}_{2}-\mathbf{D} \mathbf{x}_{1}-\mathbf{n}-\mathbf{M}_{f} \dot{\mathbf{x}}_{d 2}-\mathbf{M}_{f} \dot{\alpha}_{1}
\end{aligned}
$$

with matrix arguments ignored for readability. The final Lyapunov function can now be defined as

$$
V_{2}:=V_{1}+\frac{1}{2} \mathbf{z}_{2}^{\top} \mathbf{M}_{f} \mathbf{z}_{2}
$$

and hence

$$
\begin{aligned}
\dot{V}_{2}=\dot{V}_{1}+\mathbf{z}_{2}^{\top}\left[\mathbf{U}+\mathbf{W}-\mathbf{C} \mathbf{x}_{2}-\right. & \mathbf{D} \mathbf{x}_{1}-\mathbf{n} \\
& \left.-\mathbf{M}_{f} \dot{\mathbf{x}}_{d 2}-\mathbf{M}_{f} \dot{\alpha}_{1}\right] .
\end{aligned}
$$

Assume that the leader spacecraft is perfectly controlled in its orbit, so that $\tau_{a l}^{l b}=-\tau_{d l}^{l b}$ and $\mathbf{f}_{a l}=-\mathbf{f}_{d l}$. Assume also that the follower perturbation vectors are zero; $\mathbf{w}_{f}=\left[\mathbf{f}_{d f}^{\top},\left(\tau_{d f}^{f b}\right)^{\top}\right]^{\top}=\mathbf{0}$. Then, choosing the actuator torque $\mathbf{u}_{f}=\left[\mathbf{f}_{a f}^{\top},\left(\tau_{a f}^{f b}\right)^{\top}\right]^{\top}$ as

$$
\mathbf{u}_{f}=\mathbf{C} \mathbf{x}_{2}+\mathbf{D} \mathbf{x}_{1}+\mathbf{n}+\mathbf{M}_{f} \dot{\mathbf{x}}_{d 2}+\mathbf{M}_{f} \dot{\alpha}_{1}-\mathbf{K}_{2} \mathbf{z}_{2}-\mathbf{z}_{1}
$$

where $\mathbf{K}_{2}=\mathbf{K}_{2}^{\top}>0$, results in

$$
\dot{V}_{2}=-\mathbf{z}_{0}^{\top} \mathbf{K}_{0} \mathbf{z}_{0}-\mathbf{z}_{1}^{\top} \mathbf{K}_{1} \mathbf{z}_{1}-\mathbf{z}_{2}^{\top} \mathbf{K}_{2} \mathbf{z}_{2}
$$

and the total closed-loop subsystem dynamics

$$
\begin{aligned}
\dot{\mathbf{z}}_{0} & =-\mathbf{K}_{0} \mathbf{z}_{0}+\mathbf{z}_{1} \\
\dot{\mathbf{z}}_{1} & =-\mathbf{K}_{1} \mathbf{z}_{1}-\mathbf{z}_{0}+\mathbf{z}_{2} \\
\mathbf{M}_{f} \dot{\mathbf{z}}_{2} & =-\mathbf{K}_{2} \mathbf{z}_{2}-\mathbf{z}_{1} .
\end{aligned}
$$

The PID+ properties of the controller follows from (60) and the augmented closed loop system (62)-(64).

\subsection{Stability properties}

The stability properties of the closed loop system given by (63)-(64) follows from (58) and (61). From (58) it is seen that $V_{2}(\mathbf{z})$ is positive definite and decresent, and $V_{2}=\frac{1}{2} \mathbf{z}^{\top} \mathbf{P z}$ with $\mathbf{z}=\left[\mathbf{z}_{0}^{\top}, \mathbf{z}_{1}^{\top}, \mathbf{z}_{2}^{\top}\right]^{\top}$ and $\mathbf{P}=\operatorname{diag}\left(\mathbf{I}, \mathbf{I}, \mathbf{M}_{f}\right)$. Similarly, from (61) it is seen that $\dot{V}_{2}$ is negative definite, and $\dot{V}_{2}=-\mathbf{z}^{\top} \mathbf{Q z}$ with $\mathbf{Q}=\operatorname{diag}\left(\mathbf{K}_{1}, \mathbf{K}_{2}, \mathbf{K}_{3}\right)$. Hence, from invoking standard Lyapunov theorems ( $c f$. Khalil (2002)), it follows that both of the equilibrium points $\tilde{\mathbf{x}}_{1}=\left[\mathbf{0}^{\top} \pm 1 \mathbf{0}^{\top}\right]^{\top}$ are uniformly exponentially stable (UES), and it follows from (36) that $\mathbf{x}_{1}(t) \rightarrow \mathbf{x}_{d 1}(t)$ exponentially as $t \rightarrow \infty$. Also, (50) and (53) implies that $\tilde{\mathbf{x}}_{2}(t) \rightarrow \mathbf{0}$ as $t \rightarrow \infty$.

\subsection{Constant perturbations}

Assume now that orbital perturbations $\tau_{d f}^{f b}$ and $\mathbf{f}_{d f}$ are constant, but unknown. The control law (60) will then result in the closed-loop system (error dynamics) given by (62), (63) and

$$
\mathbf{M}_{f} \dot{\mathbf{z}}_{2}=-\mathbf{K}_{2} \mathbf{z}_{2}-\mathbf{z}_{1}+\mathbf{w}_{f} .
$$

Accordingly, in steady-state where $\dot{\mathbf{z}}=\mathbf{0}$, we have $\mathbf{z}_{1}=\mathbf{K}_{0} \mathbf{z}_{0}=\mathbf{G e}-\alpha_{0}=\mathbf{G e}+\mathbf{K}_{0} \mathbf{z}_{0} \Rightarrow \mathbf{G e}=\mathbf{0}$, which means that both of the equilibrium points $\tilde{\mathbf{x}}_{1}=\left[\mathbf{0}^{\top} \pm\right.$ $\left.1 \mathbf{0}^{\top}\right]^{\top}$ are still UES, but will converge to

$$
\mathbf{z}=\left[\begin{array}{ccc}
\mathbf{K}_{0} & -\mathbf{I} & \mathbf{0} \\
\mathbf{I} & \mathbf{K}_{1} & -\mathbf{I} \\
\mathbf{0} & \mathbf{I} & \mathbf{K}_{2}
\end{array}\right]^{-1}\left[\begin{array}{c}
\mathbf{0} \\
\mathbf{0} \\
\mathbf{w}_{f}
\end{array}\right]
$$

which are the equilibrium points of the error dynamics (cf. Fossen (2002)). Assuming that the perturbations are bounded, there exists a $\beta_{w}>0$ such that $\left\|\mathbf{w}_{f}\right\| \leq$ $\beta_{w}$, and performing the same Lyapunov analysis as above results in

$$
\dot{V}_{2}=-\mathbf{z}^{\top} \mathbf{Q} \mathbf{z}+\mathbf{z}_{2}^{\top} \mathbf{w}_{f} \leq-q_{m}\|\mathbf{z}\|^{2}+\beta_{w}\|\mathbf{z}\|
$$

where $q_{m}>0$ is the smallest eigenvalue of $\mathbf{Q}$. Accordingly, $\dot{V}_{2}<0$ when $\|\mathbf{z}\|>\delta=\beta_{w} / q_{m}$. Note also that contrary to $q_{m}, \beta_{w}$ is independent of the controller gains, so increasing the gains arbitrarily will arbitrarily diminish $\delta$. Hence, the perturbed system is uniformly practically asymptotically stable, as defined in Kristiansen et al. (2006a). From (66) it is seen that for constant gains, the steady-state errors are dependent on the perturbation term $\mathbf{w}_{f}$ only. Accordingly, for increasingly large perturbations, the steady-state errors will also increase. However, since the unperturbed system is UES, the closed-loop system will have a high degree of robustness.

\section{SIMULATIONS}

In this section, simulation results are presented to illustrate the performance of the presented control law. In the simulations, the orbital perturbations are set to $\tau_{d f}^{f b}=[-1.5,-2.5,0.011] \cdot 10^{-2}$ and $\mathbf{f}_{d f}=$ $[-13.7,1,1] \cdot 10^{-3}$. Both the leader and the follower spacecraft have masses $100 \mathrm{~kg}$, and moments of inertia given as $\mathbf{I}=\operatorname{diag}\left\{\begin{array}{lll}4.350 & 4.3370 & 3.6640\end{array}\right\} \mathrm{kgm}^{2}$. The leader spacecraft is assumed to follow an equatorial orbit with a perigee altitude of $250 \mathrm{~km}$ and eccentricity $e=0.3$. The follower spacecraft is assumed to have available continuous actuation in/about all body axes, with a maximum force and torque of $1 \mathrm{~N}$ and $0.1 \mathrm{Nm}$, respectively, and the controller gains $\mathbf{K}_{0}=\mathbf{K}_{2}=0.1 \mathrm{I}$ and $\mathbf{K}_{1}=20 \mathbf{I}$ have been used.

The initial relative positions and attitudes are standstill at $[0,-10,0] \mathrm{m}$ and $\left[-75^{\circ},-175^{\circ}, 70^{\circ}\right]$, respectively. The latter corresponds to the quaternion values $[-0.3772,-0.4329,0.6645,0.4783]$. The follower spacecraft is commanded to follow smooth sinusoidal trajectories around the origin with velocity and angular velocity profiles 

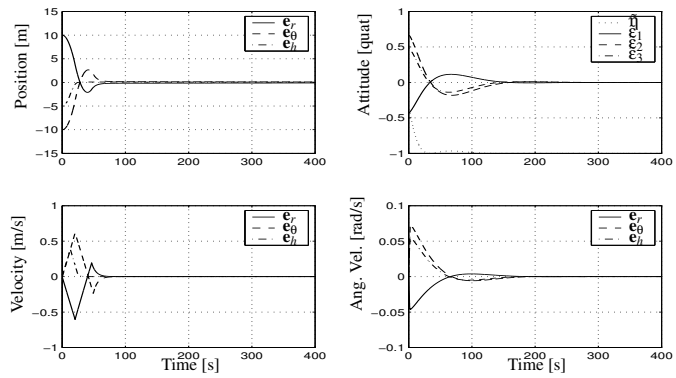

Fig. 1. Relative position, velocity, attitude and angular velocity in the settling phase
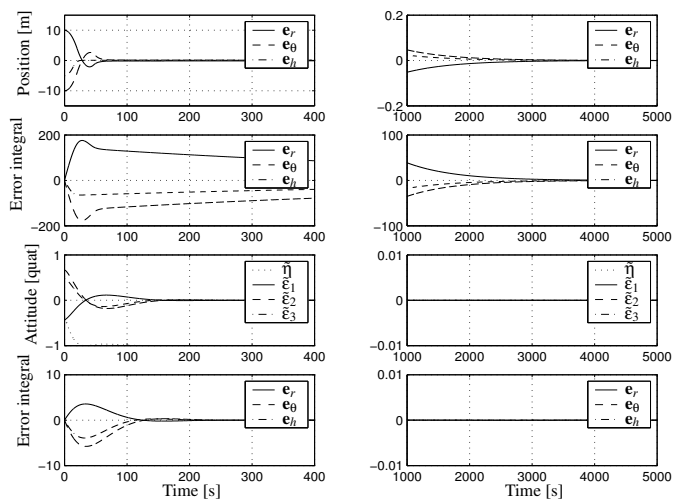

Fig. 2. Relative position, attitude and corresponding error integrals over one orbit

$$
\begin{aligned}
\mathbf{v}_{*}(t) & =\left[10 c_{o} \sin \left(c_{o} t\right), 20 c_{o} \cos \left(2 c_{o} t\right),-15 c_{o} \sin \left(3 c_{o} t\right)\right]^{\top} \\
\omega_{*}(t) & =\left[-c_{o} \sin \left(2 c_{o} t\right), \frac{8}{5} c_{o} \sin \left(4 c_{o} t\right), \frac{4}{5} c_{o} \sin \left(2 c_{o} t\right)\right]^{\top}
\end{aligned}
$$

where $c_{o}=\frac{\pi}{T_{o}}$ is a leader orbital period constant. A possible scenario for this motion is in-orbit inspection, where the follower moves in orbit around the leader.

\subsection{Results}

Fig. 1 shows the convergence of relative translation and rotation to the desired trajectories, and the convergence of the same over one orbit together with error integrals are shown in Fig. 2. As shown, both relative translation and rotation converge exponentially to the reference trajectories. Note that the relative attitude converges to the negative quaternion $-\mathbf{q}_{d}$, which from our definition in (36) is a valid equilibrium point. Note also that the perturbations in the simulations are approximately ten times larger than expected values $\left(\left|\mathbf{w}_{f}\right| \lesssim 1 \cdot 10^{-3}\right)$. For perturbations of the expected magnitude, and control gains as given above, the system will experience steady-state errors smaller than approximately $4 \cdot 10^{-3}$.

\section{CONCLUSION}

We have presented a PID+ tracking controller for relative translation and rotation in a leader-follower spacecraft formation. The result is derived using integrator backstepping, and rest on an assumption of constant, but unknown, orbital perturbations. By using an integrator augmentation technique, we included integral effect in the controller to withstand unknown orbital perturbations. The equilibrium points in the closedloop system were proved to be UES, and the solution utilizes the quaternion representation to achieve a shorter rotation path on commanded attitude changes.

\section{REFERENCES}

R. H. Battin. An Introduction to the Mathematics and Methods of Astrodynamics, Revised Edition. AIAA Education Series. AIAA, Reston, VA, 1999.

W. H. Clohessy and R. S. Wiltshire. Terminal guidance system for satellite rendezvous. Journal of Aerospace Sciences, 27(9):653-658, 1960.

O. Egeland and J. T. Gravdahl. Modeling and Simulation for Automatic Control. Marine Cybernetics, Trondheim, Norway, 2002.

T. I. Fossen. Marine Control Systems. Marine Cybernetics, Trondheim, Norway, 2002.

G. W. Hill. Researches in the lunar theory. American Journal of Mathematics, 1(1):5-26, 1878.

H. K. Khalil. Nonlinear Systems, third edition. Pearson Education International Inc., Upper Saddle River, New Jersey, USA, 2002.

R. Kristiansen, A. Loría, A. Chaillet, and P. J. Nicklasson. Output feedback control of relative translation in a leader-follower spacecraft formation. In Pettersen, Gravdahl, and Nijmeijer, editors, Group Coordination and Cooperative Control, vol. 336 of LNCIS, pp. 131-151. Springer Verlag, 2006a.

R. Kristiansen, P. J. Nicklasson, and J. T. Gravdahl. Quaternion-based backstepping control of relative attitude in a spacecraft formation. In Proc. of the 45th IEEE Conf. on Decision and Control, San Diego, CA, 2006b.

R. Kristiansen, E. I. Grøtli, P. J. Nicklasson, and J. T. Gravdahl. A model of relative translation and rotation in leader-follower spacecraft formations. Mod., Identification and Control, 28(1):3-14, 2007.

H. Pan and V. Kapila. Adaptive nonlinear control for spacecraft formation flying with coupled translational and attitude dynamics. In Proc. of the Conf. on Decision and Control, Orlando, FL, 2001.

P. K. C. Wang and F. Y. Hadaegh. Coordination and control of multiple microspacecraft moving in formation. Journal of the Astronautical Sciences, 44(3):315-355, 1996.

P. K. C. Wang, F. Y. Hadaegh, and K. Lau. Synchronized formation rotation and attitude control of multiple free-flying spacecraft. AIAA J. of Guidance, Control and Dynamics, 22(1):28-35, 1999.

H. Wong, H. Pan, and V. Kapila. Output feedback control for spacecraft formation flying with coupled translation and attitude dynamics. In Proc. of the American Control Conf., Portland, OR, 2005.

Q. Yan, G. Yang, V. Kapila, and M. de Queiroz. Nonlinear dynamics and adaptive control of multiple spacecraft in periodic relative orbits. In Proc. of the AAS Guid. and Cont. Conf., Breckenridge, CO, 2000. 